\title{
COMPARAÇÃO DE PROTOCOLOS DE CORRIDA PARA DETERMINAÇÃO DE DIFERENTES LIMIARES
}

COMPARISON OF PROTOCOLS OF FOOTRACE FOR DETERMINATION OF DIFFERENT THRESHOLDS

COMPARACIÓN DE PROTOCOLOS DE CARRERA PARA DETERMINACIÓN DE DIFERENTES UMBRALES

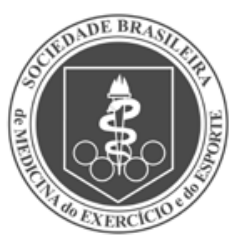

Artigo Original
Carina Helena Wasem Fraga' (Educadora Física)

Roberto Bianco' (Educador Físico) Júlio Cerca Serrão ${ }^{1}$ (Educador Físico) Camila Coelho Greco² (Educadora Física)

Alberto Carlos Amadio' (Educador Físico)

Mauro Gonçalves ${ }^{3}$ (Educador Físico)

1. Laboratório de Biomecânica da Escola de Educação Física e Esporte da Universidade de São Paulo, São Paulo, SP. Brasil.

2. Laboratório de Avaliação da Performance Humana do Departamento de Educação Física da Universidade Estadual Paulista (UNESP), Rio Claro, SP. Brasil.

3. Laboratório de Biomecânica do Departamento de Educação Física da Universidade Estadual Paulista (UNESP), Rio Claro, SP. Brasil.

\section{Correspondência:}

Escola de Educação Física e Esporte da Universidade de São Paulo. Laboratório de Biomecânica. Av. Prof. Mello Moraes, 65. São Paulo, SP, Brasil. 05508-900.

wcarina_helena@hotmail.com

\section{RESUMO}

Introdução: Testes incrementais de corrida permitem a determinação de limiares metabólicos e neuromusculares. O objetivo do presente estudo foi comparar índices eletromiográficos e metabólicos entre dois protocolos incrementais de corrida com diferentes intervalos entre cada estágio de velocidade. Métodos: Participaram do estudo 14 voluntários do sexo masculino. Os protocolos incrementais de corrida em esteira iniciaram em $8 \mathrm{~km} \cdot \mathrm{h}^{-1}$, com incremento de $1 \mathrm{~km} \cdot \mathrm{h}^{-1}$ a cada três minutos até a exaustão voluntária. Os dois protocolos diferiram quanto aos intervalos entre cada estágio de velocidade: 30 segundos (protocolo 1) e 120 segundos (protocolo 2). O limiar de fadiga eletromiográfico $\left(L F_{E M G}\right)$ foi determinado para os músculos reto femoral, bíceps femoral, tibial anterior e gastrocnêmio lateral. Para tanto, o comportamento do valor RMS foi correlacionado em função do tempo de corrida, sendo realizada regressão linear para determinação dos coeficientes de inclinação. O limiar de lactato foi identificado por meio do ponto de inflexão na curva lactato-intensidade e o limiar anaeróbio foi determinado por meio de interpolação linear. Foi aplicado um teste $t$ de Student para dados pareados $(p<0,05)$. Resultados: Foi verificado que o protocolo 2 apresentou velocidade de $L F_{E M G}$ maior do que o protocolo 1, apenas para o músculo $B F(p=0,023)$, o que caracteriza uma resposta específica deste músculo em protocolos incrementais de corrida. Conclusão: Protocolos de corrida com intervalos de até dois minutos entre os estágios incrementais apresentaram resultados semelhantes para determinação do $L F_{E M G}$ da maioria dos músculos estudados e dos limiares metabólicos.

Palavras-chave: eletromiografia, fadiga, lactato.

\begin{abstract}
Introduction: Incremental running tests allow the determination of neuromuscular and metabolic thresholds. The purpose of this study was to compare electromyographic and metabolic indexes between two incremental running protocols with different interval durations at each stage of velocity. Methods: Fourteen male subjects took part in this study. The incremental protocols consisted of treadmill running at an initial velocity of $8 \mathrm{~km} . \mathrm{h}^{-1}$, with increments of $1 \mathrm{~km} \cdot \mathrm{h}^{-1}$ every three minutes until voluntary exhaustion. The two protocols differed in their intervals between each stage of velocity: 30 seconds (protocol 1) and 120 seconds (protocol 2). The electromyographic fatigue threshold ( $E M G_{F T}$ ) was determined for the rectus femoris, biceps femoris, tibialis anterior and gastrocnemius lateralis muscles. For this purpose the behavior of the RMS value was correlated according to the running time, performing linear regression to determine the slope coefficients. The lactate threshold was calculated using the point of inflection on the lactate intensity curve and the anaerobic threshold was determined by linear interpolation. A paired student t-test was applied $(p<0.05)$. Results: Protocol 2 was seen to have higher $E M_{F F}$ values than protocol 1 only for the BF muscle $(p=0.023)$, which characterizes a specific response of this muscle in incremental running protocols. Conclusion: Running protocols with intervals of up to two minutes between incremental stages showed similar $E M G_{F T}$ results for most of the muscles and similar metabolic thresholds.
\end{abstract}

Keywords: electromyography, fatigue, lactate.

\section{RESUMEN}

Introducción: Los tests incrementales de carrera permiten la determinación de umbrales metabólicos y neuromusculares. El objetivo del presente estudio fue comparar índices electromiográficos y metabólicos entre dos protocolos incrementales de carrera con diferentes intervalos entre cada etapa de velocidad. Métodos: Participaron en el estudio 14 voluntarios de sexo masculino. Los protocolos incrementales de carrera en cinta de correr se iniciaron en $8 \mathrm{~km} \cdot h^{-1}$, con incremento de $1 \mathrm{~km} \cdot h^{-1}$ a cada tres minutos hasta el agotamiento voluntario. Los dos protocolos diferiron cuanto a los intervalos entre cada etapa de velocidad: 30 segundos (protocolo 1) y 120 segundos (protocolo 2). El umbral de fatiga electromiográfico ( $\left.L F_{E M G}\right)$ fue determinado para los músculos recto femoral, biceps femoral, tibial anterior y gastrocnemio lateral. Para tanto, el comportamiento del valor RMS fue correlacionado en función del tiempo de carrera, siendo realizada regresión lineal para determinación de los coeficientes de inclinación. El umbral de lactato fue identificado por medio del punto de inflexión en la curva lactato-intensidad y el umbral anaeróbico fue determinado por medio de interpolación lineal. Fue aplicado un test t de Student para datos apareados $(p<0,05)$. Resultados: Fue verificado que el protocolo 2 presentó velocidad 
de $L F_{E M G}$ mayor que el protocolo 1, sólo para el músculo $B F(p=0,023)$, lo que caracteriza una respuesta específica de este músculo en protocolos incrementales de carrera. Conclusión: Los protocolos de carrera con intervalos de hasta dos minutos entre las etapas incrementales presentaron resultados semejantes para determinación del $L F_{E M G}$ de la mayoría de los músculos estudiados y de los umbrales metabólicos.

Palabras clave: electromiografía, fatiga, lactato.

\section{INTRODUÇÃO}

Existe grande interesse em compreender melhor a fadiga e sua manifestação em variáveis fisiológicas e biomecânicas. Particularmente, grande atenção pode ser observada com relação à fadiga muscular, pois a melhor compreensão deste fenômeno possibilita maior controle sobre o risco do surgimento de lesões e melhor direcionamento das estratégias de treinamento. A fadiga muscular é caracterizada por uma gradual redução na capacidade de produção de força, que pode ser associada a alterações na atividade eletromiográfica ou à exaustão da função contrátil'.

Para compreender a relação das variáveis fisiológicas e biomecânicas com a fadiga muscular, é necessário induzir diferentes níveis de fadiga no organismo de forma controlada até a exaustão e medir o comportamento do aparelho locomotor durante esse processo ${ }^{2,3}$. Neste sentido, testes incrementais de corrida em esteira são interessantes, na medida em que permitem a análise de indicadores biomecânicos e metabólicos em situações de indução à fadiga controlada 4 . Limiares obtidos em testes incrementais são frequentemente utilizados para avaliar a resposta do lactato sanguíneo em função da modificação da intensidade de exercício ${ }^{5,6}$.

De acordo com Hue et al.', a utilização dos valores de limiar como ponto de referência possibilita expressar de forma mais precisa as intensidades de exercícios submáximos. É bastante comum o uso do limiar de lactato para esse fim, mas ainda não está bem determinada a relação que o Limiar de Fadiga Eletromiográfico $\left(\mathrm{LF}_{\mathrm{EMG}}\right)$ apresenta com as diferentes intensidades de exercício. O LFF é um indicador da mais alta intensidade de exercício sustentável sem evidência de fadiga neuromuscular. Nas intensidades abaixo do $L F_{E M G}$ a atividade eletromiográfica não se altera ao longo do tempo e, desta forma, exercícios podem ser mantidos por um período prolongado sem exaustão $0^{8,9}$.

Testes incrementais de corrida pressupõem aumentos progressivos de intensidade até a exaustão e, frequentemente, essa variação na intensidade é realizada por meio de alterações na velocidade de movimento. Entretanto, os diferentes protocolos de testes apresentam variação na forma como a intensidade é incrementada. Alguns protocolos manipulam a duração de cada estágio incremental, a inclinação ou os intervalos entre cada estágio incremental. Na literatura verificam-se estudos que compararam protocolos com diferentes durações dos estágios de corrida incremental ${ }^{5,10}$, e protocolos com incrementos distintos - velocidade e inclinação da esteira ${ }^{11}$. No entanto, faz-se necessária a realização de estudos que comparem os efeitos de diferentes intervalos entre os estágios de velocidade de protocolos incrementais de corrida na determinação de limiares. Para esse fim, geralmente são utilizados protocolos com intervalos menores do que 30 segundos entre os estágios incrementais. Este intervalo apresenta-se como o tempo mínimo necessário para a coleta de amostras sanguíneas, ${ }^{512-13}$. Se os limiares metabólicos e neuromusculares não se alterarem em protocolos com maiores intervalos entre os estágios incrementais, haverá a possibilidade de aquisição de dados adicionais como, por exemplo, a avaliação do controle postural, determinação de força submáxima e da ativação muscular, entre outros dados biomecânicos e fisiológicos. Dessa forma, tais coletas adicionais podem contribuir para o entendimento dos efeitos da fadiga sobre o controle do movimento pelo aparelho locomotor.
Diante do exposto, o objetivo do presente estudo foi comparar os efeitos de dois protocolos incrementais de corrida em esteira rolante, que utilizaram diferentes intervalos de tempo entre os estágios de velocidade do teste, para determinação de limiares eletromiográficos e metabólicos.

\section{MATERIAL E MÉTODOS}

Participaram deste estudo 14 voluntários do sexo masculino (idade média de 20,4 \pm 1,64 anos, massa corporal de 68,56 $\pm 7,78 \mathrm{~kg}$, estatura de 1,75 $\pm 0,03 \mathrm{~m}$ e percentual de gordura de 13,3 $\pm 3,82$ ), sem antecedentes de lesões nos membros inferiores e com antropometria semeIhante. Os voluntários treinavam para diferentes modalidades esportivas há, pelo menos, dois anos, sendo que todos utilizavam a corrida como forma de treinamento aeróbio. Os voluntários avaliados e seus técnicos foram cuidadosamente orientados sobre o experimento, e assinaram um termo de consentimento livre e esclarecido, concordando com sua participação voluntária. O presente estudo foi submetido e aprovado pelo Comitê de Ética em Pesquisa local (Protocolo 2771/2004).

\section{Protocolo de teste}

Inicialmente, os sujeitos foram submetidos a uma anamnese ortopédica para garantir a integridade do aparelho locomotor. Em seguida, foram obtidas medidas antropométricas, na tentativa de melhor caracterizar a amostra deste estudo. Todos os voluntários foram submetidos a um protocolo de familiarização da corrida na esteira. Esta familiarização consistiu de nove minutos de corrida, divididos em três estágios de 3 minutos. Em cada estágio, foram usadas intensidades progressivamente maiores correspondentes às velocidades: 8,9 e $10 \mathrm{~km} \cdot \mathrm{h}^{-1}$. A familiarização visou minimizar o efeito de aprendizagem do teste, que poderia interferir nos resultados do estudo. Para reduzir a interferência da familiarização, foi oferecido um intervalo de, no mínimo, 72 horas entre a familiarização e a realização do teste incremental.

A partir da familiarização, duas visitas ao laboratório foram agendadas para cada sujeito, a fim de realizar os dois protocolos de teste em dias diferentes. Nos dois protocolos, a velocidade inicial foi de $8 \mathrm{~km} \cdot \mathrm{h}^{-1}$, cada estágio de teste tinha a duração de 3 minutos e, ao término de cada estágio, havia um incremento na velocidade de $1 \mathrm{~km} \cdot \mathrm{h}^{-1}$ a cada 3 minutos até a interrupção do teste. O critério usado para a interrupção do teste foi a exaustão voluntária do sujeito, definida como o momento quando o voluntário não fosse capaz de manter a velocidade determinada. A diferença entre os protocolos reside na duração dos intervalos estabelecidos entre cada estágio de teste: intervalo de 30 segundos (protocolo 1); intervalo de 120 segundos (protocolo 2).

A inclinação da esteira foi mantida inalterada em 1\%, já que esta condição reflete mais precisamente o custo energético da corrida em piso fixo, em ambientes abertos ${ }^{14}$. A ordem de realização dos protocolos foi randomizada. Todas as avaliações foram realizadas com, pelo menos, 72 horas de intervalo entre elas, na tentativa de evitar que a fadiga pudesse interferir nos resultados obtidos. Os sinais EMG foram coletados em cada estágio de velocidade, durante todo o teste incremental. As amostras de $25 \mu$ l de sangue do lóbulo da orelha foram coletadas ao final de cada estágio de velocidade de corrida, durante os intervalos (30 ou 120 segundos). Para a coleta de dados, foi usada uma esteira rolante 
motorizada (INBRAMED SUPER ATL, Brasil), com dimensões de $2 \mathrm{~m}$ de comprimento e $63 \mathrm{~cm}$ de largura. Para a captação dos sinais EMG foi utilizado o sistema Telemyo 900 (Noraxon) e software Myoresearch (Noraxon).

\section{Aquisição e análise dos dados eletromiográficos}

Os sinais EMG foram adquiridos por meio de eletrodos de superfície descartáveis (Ag/AgCl), em configuração bipolar. Os eletrodos foram posicionados do lado direito do corpo, nos músculos reto femoral (RF), bíceps femoral (BF), gastrocnêmio lateral (GL) e tibial anterior (TA), de acordo com as normas sugeridas no SENIAM ${ }^{15}$. Já o eletrodo de referência foi posicionado sobre a da face anterior da tíbia, também do lado direito do corpo.

Previamente à colocação dos eletrodos, foram realizados os procedimentos de tricotomia e limpeza da pele com lixa fina e álcool, no intuito de diminuir possíveis interferências na aquisição do sinal EMG.

A frequência de amostragem utilizada foi de $1000 \mathrm{~Hz}$, com ganho de 2000 vezes (20 vezes no pré-amplificador e 100 vezes no condicionador de sinais). Os dados brutos foram filtrados com filtros: notch $60 \mathrm{~Hz}$, passa alta de $20 \mathrm{~Hz}$ e passa baixa de $500 \mathrm{~Hz}$. Posteriormente, o sinal filtrado foi retificado.

Para análise dos dados eletromiográficos, foi utilizada uma rotina desenvolvida em ambiente MatLab (versão 6.5). Os sinais EMG foram analisados no domínio do tempo, por meio do cálculo da amplitude do sinal EMG, expresso pelo valor RMS (Root Mean Square).

\section{Aquisição e análise das amostras sanguíneas}

Previamente à coleta de sangue, foi realizada assepsia local com álcool e algodão e a punção do lóbulo da orelha com uma lanceta descartável. A primeira gota de sangue foi descartada para evitar a contaminação pelo suor produzido. Foram coletados $25 \mu \mathrm{l}$ de sangue arterializado do lóbulo da orelha com capilares de vidro heparinizados e calibrados. Após cada coleta, o sangue foi imediatamente depositado em tubos tipo Eppendorf de 1,5 ml contendo $50 \mu \mathrm{l}$ de fluoreto de sódio a 1\%. O lactato sanguíneo foi determinado por um método eletroquímico (YSL 2300 STAT, Yelow Springs, Ohio, EUA).

\section{Determinação dos limiares eletromiográficos $\left(\mathrm{LF}_{\mathrm{EMG}}\right)$ e metabólicos}

$O L F_{E M G}$ foi determinado para cada músculo analisado usando os dois minutos finais de cada estágio de velocidade. Nesses intervalos de dois minutos, valores RMS em janelas de cinco segundos foram calculados, obtendo 24 valores de RMS para cada estágio de teste incremental, ou seja, para cada velocidade de corrida. A figura 1 ilustra os 24 valores de RMS para cinco velocidades de corrida distintas. Os valores de RMS foram correlacionados com o tempo e a regressão linear foi calculada para cada velocidade de corrida. A partir das correlações, os coeficientes de inclinação de cada velocidade foram obtidos.

Em seguida foi realizada nova regressão linear entre as velocidades (eixo das ordenadas) e seus respectivos coeficientes de inclinação (eixo das abscissas) para a determinação do intercepto - valor equivalente a uma velocidade de corrida em que o coeficiente de inclinação foi zero (figura 2). A velocidade que corresponde ao intercepto é o valor considerado como a velocidade do $L F_{E M G}$. Assim, o LF $F_{E M G}$ de cada músculo foi determinado pelo valor do intercepto no eixo da velocidade ${ }^{8,16}$.

O limiar de lactato (LL) foi determinado por meio do ponto de inflexão na curva lactato-intensidade (determinado por três pesquisadores experientes). O limiar anaeróbio (LAn) foi determinado por meio de interpolação linear, considerando a concentração fixa de 3,5 $\mathrm{mM}^{17}$. A figura 3 apresenta um exemplo de como o LL e LAn foram determinados para um indivíduo que compõe a amostra deste estudo.

\section{Análise dos dados}

A normalidade de distribuição dos dados foi avaliada com teste de Shapiro-Wilk. Uma vez confirmada a normalidade de distribuição

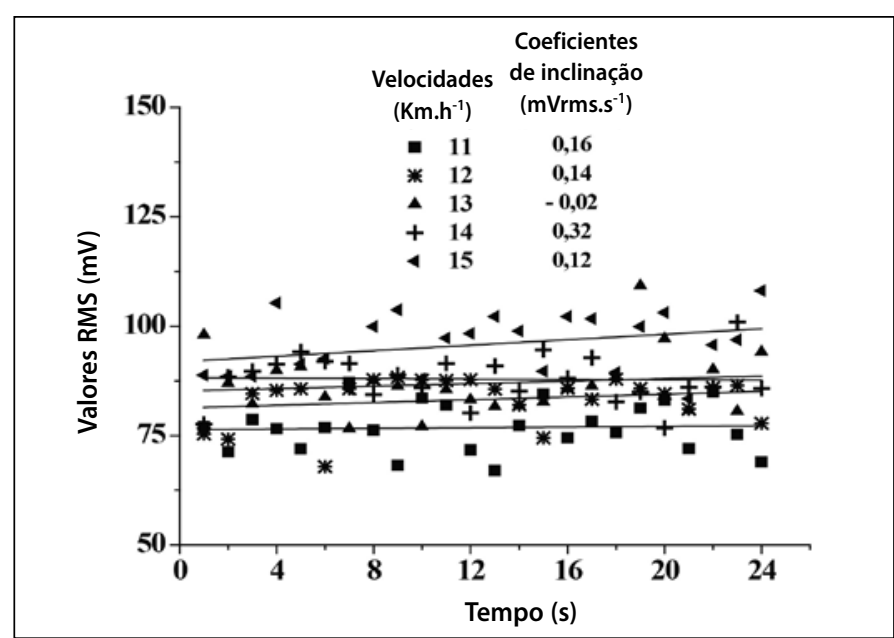

Figura 1. Distribuição dos 24 valores de RMS de cada velocidade de corrida, ao longo do tempo para um sujeito, como forma de ilustrar como o LFEMG foi obtido. Valores obtidos para um sujeito que compõe a amostra, para o músculo VL.

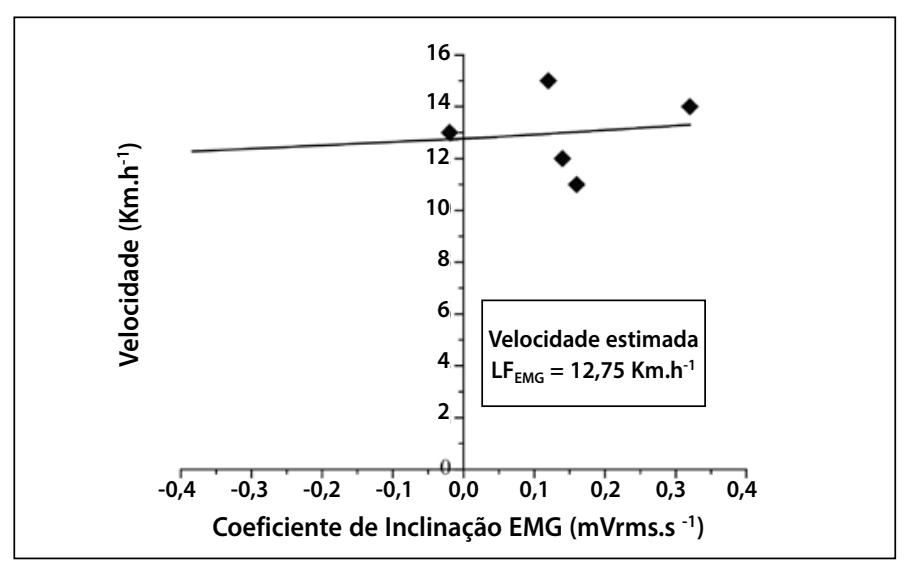

Figura 2. Coeficientes de inclinação apresentados em relação as suas respectivas velocidades de corrida para estimar o LFEMG (valor correspondente ao y-intercepto).

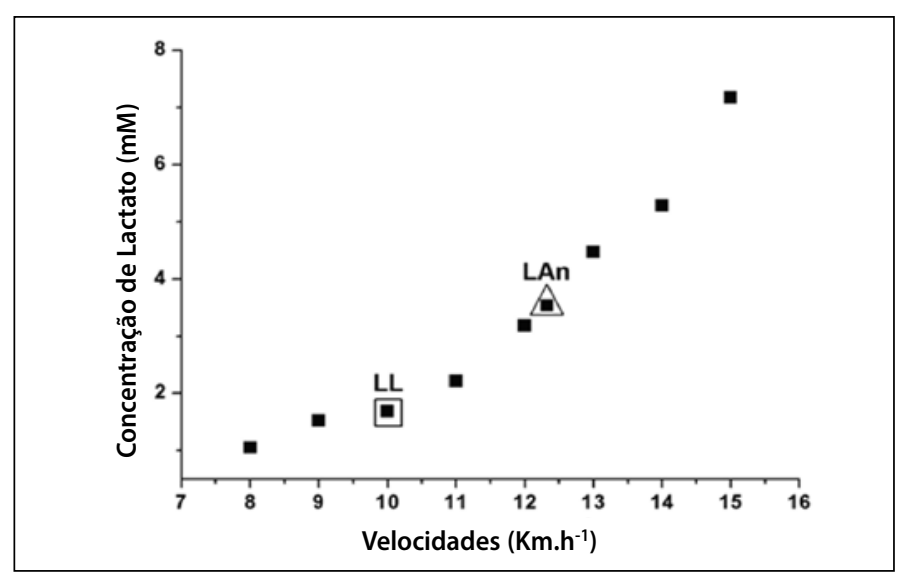

Figura 3. Exemplo da determinação dos valores de $L L$ e LAn para um sujeito da amostra deste estudo. LAn foi determinado por meio do ponto de inflexão na curva de lactato pela intensidade de corrida, (10 km.h-1: simbolizado pelo quadrado). LL foi determinado por meio de interpolação linear, considerando a concentração fixa de $3.5 \mathrm{mM}$ (12.24 km.h-1: simbolizado pelo triângulo).

dos dados, foram calculadas as médias e desvios padrão para todas as variáveis analisadas. Para comparação dos diferentes limiares entre os dois protocolos de teste foi aplicado um teste $t$ de Student para dados pareados. Foi considerado, para todos os cálculos, $p<0,05$. Para a realização do tratamento estatístico foi utilizado o aplicativo SPSS (Statistical Package for Social Sciences), versão 13.0. 


\section{RESULTADOS}

As velocidades máximas atingidas pelo grupo avaliado em cada protocolo (1 e 2), expressas em médias e desvios-padrão, foram 14,86 $\pm 1,10 \mathrm{~km} \cdot \mathrm{h}^{-1}$ e 15,50 $\pm 1,34 \mathrm{~km} \cdot \mathrm{h}^{-1}$, respectivamente.

A Figura 4 apresenta as velocidades correspondentes ao $L F_{E M G}$ determinado para os diferentes músculos e mostra a comparação desses limiares entre os protocolos 1 e 2.

De acordo com a análise da figura 4, é possível verificar diferença significativa apenas para o músculo BF $(p=0,023)$. Na Figura 5 são apresentados os dados referentes à comparação entre os dois protocolos (Protocolo 1 e 2) utilizados para determinação dos limiares metabólicos (LL e LAn). Não foram verificadas diferenças significativas entre os dois protocolos utilizados para determinação dos limiares metabólicos.

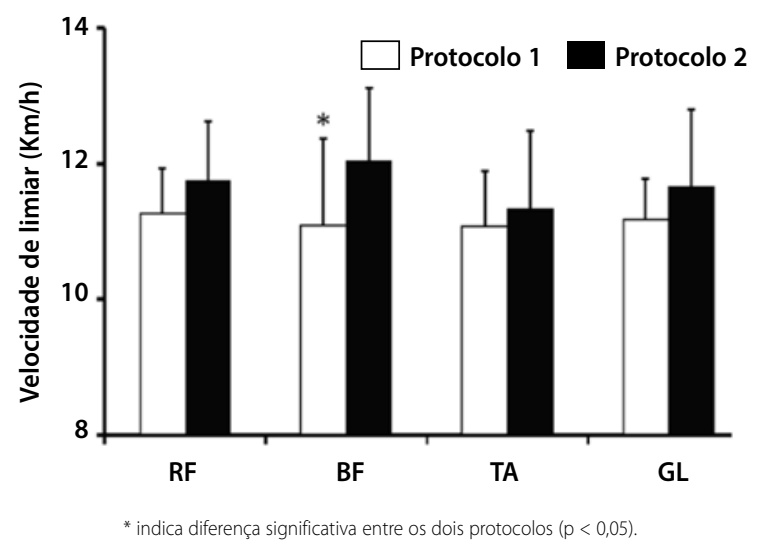

Figura 4. Velocidades (km.h-1) correspondentes ao LFEMG determinado para os músculos reto femoral (RF), bíceps femoral porção lateral (BF), tibial anterior (TA) e gastrocnêmio porção lateral (GL).

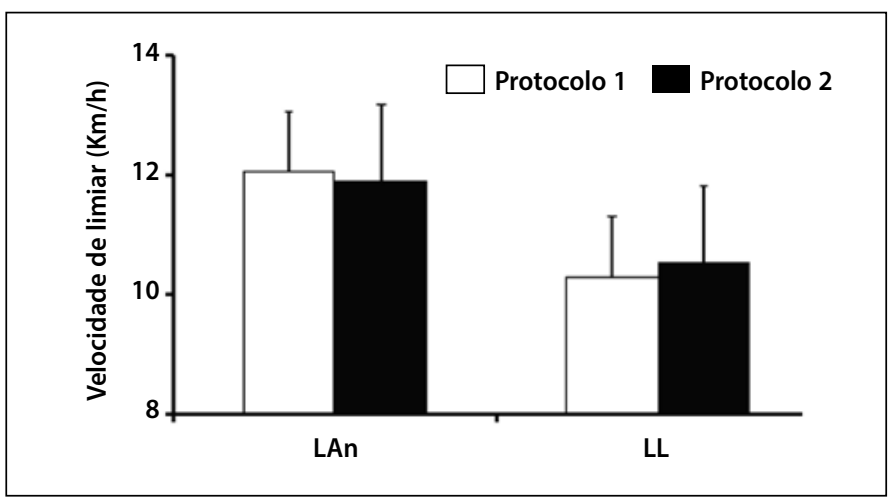

Figura 5. Velocidades de corrida (km.h-1) correspondentes ao LL e LAn.

\section{DISCUSSÃO}

O presente estudo procurou comparar índices eletromiográficos e metabólicos obtidos a partir de diferentes protocolos de corrida incremental, os quais diferiram de acordo com os intervalos estabelecidos entre cada estágio de velocidade: intervalo de 30 segundos; intervalo de 120 segundos. Foi verificada diferença na velocidade correspondente ao $L_{E M G}$ apenas para o músculo BF. Não foram encontradas diferenças para os demais músculos analisados e para o LAn e o LL.

A utilização do $L F_{\text {EMG }}$ para a estimativa da fadiga neuromuscular fornece uma importante alternativa para a determinação da mais alta intensidade de exercício que pode ser sustentada sem causar fadiga, $8,16.18$. $O$ protocolo 2 apresentou velocidade de $\mathrm{LF}_{\mathrm{EMG}}$ significativamente maior do que o protocolo 1 para o músculo $B F$, ou seja, o $L F_{E M G}$ do músculo BF ocorreu em uma velocidade mais alta no protocolo 2 do que no protocolo 1. Nos demais músculos, embora as médias aparentem ser maiores, as diferenças entre os protocolos não foram significativas. Isso pode indicar que mesmo intervalos de tempo relativamente curtos entre os estágios, por exemplo, 30 segundos, podem promover certo grau de recuperação nesses músculos. $O$ fato de apenas o LF $F_{E M G}$ do músculo BF ter se apresentado diferente entre os protocolos, pode ter ocorrido devido ao intervalo específico imposto entre os estágios. É possível que intervalos maiores de recuperação promovam diferenças em outros músculos, e não apenas no BF.

Além disso, especula-se que o menor valor de $L_{E M G}$ para o BF possa estar associado a algumas características biomecânicas específicas deste músculo. Como o músculo BF é biarticular, ele apresenta funções específicas nas articulações do quadril e joelho durante cada ciclo de passada da corrida. Na segunda metade da fase de balanço o músculo se encontra ativado em contração excêntrica para desacelerar o movimento de flexão do quadril e de extensão do joelho. Similar ao músculo RF, nesta fase o músculo BF desempenha um importante papel na transferência de energia entre as articulações. Já na fase de contato, o BF encontra-se em ativação para realizar o movimento de extensão do quadril em contração concêntrica ${ }^{19}$. Outro aspecto a ser considerado é que alguns estudos levantam a possibilidade de que existam diferenças na atuação de músculos mono e biarticulares para a produção do movimento. Tem sido demonstrado que músculos monoarticulares desempenham importante papel na produção de movimento na articulação do joelho, enquanto que músculos biarticulares, como o BF, parecem fornecer ajustes refinados no controle da distribuição dos torques produzidos nestas articulações ${ }^{20,21}$. Obviamente, também é possível que as diferenças específicas no BF sejam consequência de características individuais dos sujeitos avaliados neste estudo ${ }^{22}$.

Um estudo realizado por Gazendam e Hof $^{23}$ permitiu verificar que na corrida (assim como na marcha) a análise da ativação de um grupo muscular pode ser feita usando um músculo representante desse grupo com funções comuns, pois os ventres desse grupo muscular apresentam padrões de sinal eletromiográfico semelhantes: (1) grupo do quadríceps (2) grupo dos ísquiotibiais (3) grupo do tríceps sural; (4) grupo dos glúteos. Neste mesmo estudo, foi verificado que em testes incrementais de corrida não há um comportamento estável do sinal eletromiográfico associado ao incremento de velocidade. Isso significa que o sinal eletromiográfico pode aumentar, permanecer constante ou, até mesmo, diminuir a partir do incremento de velocidade, dependendo do músculo a ser analisado. Em geral, existe uma tendência de aumento do sinal eletromiográfico, a partir do incremento de velocidade de corrida, em função do recrutamento de unidades motoras que aumentam seu disparo pela maior necessidade de estabilidade articular e, também, em função da instalação de um processo de fadiga ${ }^{24,25}$. Contudo, no estudo realizado por Gazendam e Hof ${ }^{19}$ os autores observaram que a amplitude do sinal eletromiográfico do músculo BF apresentou uma resposta diferenciada a partir do incremento de velocidade. De acordo com os resultados encontrados por estes autores, foi verificado aumento da amplitude do sinal EMG para o músculo BF com o incremento de velocidade de corrida até a velocidade de $3,0 \mathrm{~m} \cdot \mathrm{s}^{-1}$ e redução da amplitude após essa velocidade até 4,5 m.s. ${ }^{-1}$. Especula-se que estas características específicas no perfil de ativação do músculo BF a partir da realização protocolos incrementais possam, também, ser uma possível explicação para as diferenças encontradas no $L_{E M G}$ para este músculo em particular.

A utilização de limiares metabólicos, como o LL e o LAn como forma de demarcar intensidades de exercício que auxiliem na prescrição e elaboração de programas de treinamento tem sido amplamente descrita na literatura ${ }^{26.27}$. Não foram verificadas diferenças significativas nos limiares metabólicos (LL e LAn) entre os dois protocolos propostos. 
Com isso, foi possível constatar que intervalos de até dois minutos, em protocolos incrementais de corrida em esteira não apresentaram interferência na determinação dos limiares metabólicos para os sujeitos avaliados. Possíveis diferenças encontradas na análise da fadiga muscular durante a corrida podem ser explicadas por diversos fatores como experiência do sujeito na modalidade, duração e distância da corrida, intensidade de teste, e protocolo de teste ${ }^{28}$. Assim, especula-se que esta resposta metabólica esteja associada ao nível de treinamento dos sujeitos avaliados e, que em atletas altamente treinados, essa resposta seja distinta. Outro fator relevante é que ambos os protocolos incrementais utilizam a corrida como forma de exercício, promovendo estímulos muito próximos, o que pode ser o motivo da ausência de diferenças significativas entre os limiares metabólicos ${ }^{11}$.

Os $L F_{E M G}$ dos músculos investigados no presente estudo ocorreram, para todos os músculos, em velocidades entre o LL e o LAn, no protocolo 1. Já no protocolo 2, a velocidade do $L_{E_{E M G}}$ ocorreu entre os dois limiares, na maioria dos músculos, mas não em todos. Isso denota que, no presente estudo, ainda em fase anaeróbia compensada, os músculos apresentaram indícios de que a fadiga já começou a se instalar. Essa diferença pode ter ocorrido por conta de diferenças no protocolo empregado, como intensidade e incrementos nos estágios do protocolo, ou características diferentes no grupo analisado com relação ao nível de condicionamento dos sujeitos da amostra.

Taylor e Bronks ${ }^{29}$ verificaram que o aumento não linear dos valores da integral do sinal eletromiográfico tem sido relacionado à ocorrência da transição das fases aeróbias-anaeróbias durante testes realizados em cicloergômetros. Esses resultados podem indicar que o sinal eletromiográfico pode ser utilizado como uma importante ferramenta para determinação não-invasiva do limiar de lactato.

No intuito de verificar o efeito da fadiga por meio da análise do sinal EMG em um protocolo incremental de corrida, Hanon et al. ${ }^{18}$ caracterizaram o início da fadiga neuromuscular pelo aumento da amplitude do sinal. Neste estudo foram verificados que o limiar eletromiográfico foi determinado na velocidade de $16 \mathrm{~km} . \mathrm{h}^{-1}$, enquanto que o limiar de lactato e o limiar anaeróbio foram determinados nas velocidades de $14 \mathrm{~km} \cdot \mathrm{h}^{-1}$ e 15,71 km. $\mathrm{h}^{-1}$, respectivamente. Dessa forma, o limiar eletromiográfico coincidiu com o limiar anaeróbio.

\section{REFERÊNCIAS}

1. Enoka RM, Duchateau J. Muscle fatigue: what, why and how it influences muscle function. J Physiol. 2008;1:11-23.

2. Nummela A, Rusko H, Mero A. EMG activities and ground reaction forces during fatigued and nonfatigued sprinting. Med Sci Sports Exer. 1994;26:605-9.

3. Nigg BM, Stefanyshyn D, Cole G, Stergiou P, Miller J. The effect of material characteristics of shoe soles on muscle activation and energy aspects during running. J Biomech. 2003;36:569-75.

4. Silva SRD, Fraga CHW, Gonçalves M. Efeito da fadiga muscular na biomecânica da corrida: uma revisão. Rev Motriz. 2007;13:225-35.

5. Kuipers H, Rietjens G, Verstappen F, Schoenmarkers H, Hofman G. Effects of stage duration in incremental running tests on physiological variables. Int J Sports Med. 2003;24:486-91.

6. Kohrt WM; O'Connor JS; Skinner JS. Longitudinal assessment of responses by triathletes to swimming, cycling, and running. Med Sci Sports Exer. 1989;21:569-75.

7. Hue $\mathrm{O}$, Le Gallais $\mathrm{D}$, Chollet $\mathrm{D}$, Préfaut $C$. Ventilatory threshold and maximal oxygen uptake in present triathletes. Can J Appl Physiol. 2000;25:102-13.

8. Matsumoto T, Ito K, Moritani T. The relationship between anaerobic threshold and electromyographic fatigue threshold in college women. Eur J Appl Physiol Occup Physiol. 1991;63:1-5.

9. Bishop DJ. Fatigue during intermittent-sprint exercise. Clin Exp Pharmacol Physiol. 2012;39:836-41.

10. Foxdal P, Sjödin A, Sjödin B. Comparison of blood lactate concentration obtained during incremental and constant intensity exercise. Int J Sports Med. 1995;17:360-5.

11. Kang J, Chaloupka EC, Mastrangelo MA, Biren GB, Robertson RJ. Physiological comparisons among three maximal treadmill exercises protocols in trained and untrained individuals. Eur J Appl Physiol. 2001;84:291-5.

12. Carter H, Pringle JSM, Barstow TJ, Doust JH. Oxygen Uptake Kinetics During Supra $\mathrm{VO}_{2 \max }$ Treadmill Running in Humans. Int J Sports Med. 2006;27:149-57.

13. Carter H, Pringle JSM, Jones AM; Doust JH. Oxygen uptake kinetics during treadmill running across exercise intensity domains. Eur J Appl Physiol. 2002;86:347-54.

14. Jones AM, Doust JH. A $1 \%$ treadmill grade most accurately reflects the energetic cost of outdoor running. J Sport Sci. 1996;14:321-7.

15. Hermes HJ, Freriks B, Merletti R, Hägg G, Stegeman D, Blok J. et al. editors. SENIAM 8: European recommendations for surface electromyography. ISBN: 90-75452-15-2: Roessingh Research and Development bv, 1999.
A ocorrência dos $L F_{E M G}$ em velocidades mais baixas que o $L A n$ permite especular que, em velocidades inferiores ao LAn, mas acima do $L_{E M G}$ o processo de fadiga de um músculo específico poderia levar a uma piora no controle do movimento e, consequentemente, no rendimento. Portanto, identificar os limiares dos diferentes músculos permitiria a elaboração de treinamentos mais direcionados a fim de atender à necessidade especifica de um determinado músculo. Embora a ideia seja instigante, vale lembrar que a relação entre estas variáveis e a fadiga necessita ser mais bem compreendida antes de ser usada de forma consistente na periodização de treinamento de praticantes de diferentes modalidades.

Dessa forma, foi verificado que no protocolo $20 \mathrm{LF}_{\mathrm{EMG}}$ foi obtido em uma velocidade significativamente maior do que o protocolo 1, apenas para o músculo BF. Os limiares metabólicos (LL e LAn) bem como o LF $F_{E M G}$ determinado para os demais músculos estudados não diferiram de acordo com o protocolo de teste utilizado.

\section{CONCLUSÃO}

Conclui-se que protocolos de corrida em esteira com intervalos de 30 segundos entre os incrementos apresentam resultados semelhantes a protocolos com intervalos de até dois minutos entre os estágios incrementais, para a determinação do $L F_{E M G}$ da maioria dos músculos estudados e dos limiares metabólicos. Contudo, vale lembrar que esses resultados se aplicam para o grupo de sujeitos avaliados utilizando o intervalo de tempo determinado entre os estágios nos dois protocolos. Assim, não há como afirmar se estes resultados se manteriam em intervalos de tempo superiores a 120 segundos.

\section{AGRADECIMENTOS}

Os autores agradecem à Fundação de Amparo à Pesquisa do Estado de São Paulo, FAPESP (processo 2005/02535-2; 2007/58339-2) e à Coordenação de Aperfeiçoamento de Pessoal de Nível Superior, CAPES pelos auxílios concedidos.

Todos os autores declararam não haver qualquer potencial conflito de interesses referente a este artigo.

16. DeVries HA, Moritani T, Nagata A, Magnussen K. The relation between critical power and neuromuscular fatigue as estimated from electromyographic data. Ergonomics. 1982;25:783-91.

17. Heck H, Mader A, Hess G, Mucke S, Muller R; Hollmann W. Justification of the $4 \mathrm{mmol} / \mathrm{l}$ lactate threshold. Int J Sports Med. 1985;6:117-30.

18. Hanon C, Thépaut-Mathieu C, Hausswirth C, Le Chevalier JM. Electromyogram as an indicator of neuromuscular fatigue during incremental exercise. Eur J Appl Physiol Occup Physiol. 1998;78:315-23. 19. Novacheck TF. The biomechanics of running. Gait Posture. 1998; 7:77-95.

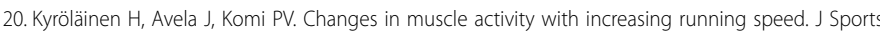
Sci. 2005;23:1101-9.

21. Van Ingen Schenau GJI, Pratt CA, Macpherson JM. Differential use and control of mono-and biarticular muscles. Human Mov Sci. 1994;13:495-517.

22. Vuorimaa T, Virlander R, Kurkilahti P, Vasankari T, Hãkkinen K. Acute changes in muscle activation and leg extension performance after different running exercises in elite long distance runners. Eur J App Physiol. 2006;96:282-91.

23. Gazendam MGJ, Hof AL. Averaged EMG profiles in jogging and running at different speeds. Gait Posture. 2007;25:604-14

24. Wakeling JM, Pascual SA, Nigg BM, Tscharner V. Surface EMG shows distinct populations of muscle activity when measured during sustained sub-maximal exercise. Eur J Appl Physiol. 2001;86:40-7.

25. Cardozo AC, Gonçalves M. Electromyographic fatigue threshold of erector spinae muscle induced by a muscular endurance test in health men. Electromyog Clin Neurophysiol. 2003;43:377-80.

26. Billat LV. Use of blood lactate measurement for prediction of exercise performance and for control of training. Sports Med. 1996;22:157-75.

27. Pollock ML, Gaesser GA, Butcher JD, Després J, Dishman RK, Franklin BA et al. The recommended quantity and quality of exercise for developing and maintaining cardiorespiratory and muscular fitness, and flexibility in healthy adults. Med Sci Sports Exer. 1998;30:975-91.

28. Saldanha A, Ekblom MMN, Thorstensson A. Central fatigue affects plantar flexor strength after prolonged running. Scand J Med Sci Sports. 2008;18:383-8.

29. Taylor AD, Bronks R. Electromyographic correlates of the transition from aerobic to anaerobic metabolism in treadmill running. Eur J Appl Physiol Occup Physiol. 1994;69:508-15. 